UCRL-ID-120947

\title{
Status Report on Source Properties Important for Discrimination
}

\author{
Lewis A. Glenn
}

This paper is submitted as

FY95 Scheduled Deliverable No. 8 for LLNL's Regional Seismic Project

\section{March 1995}

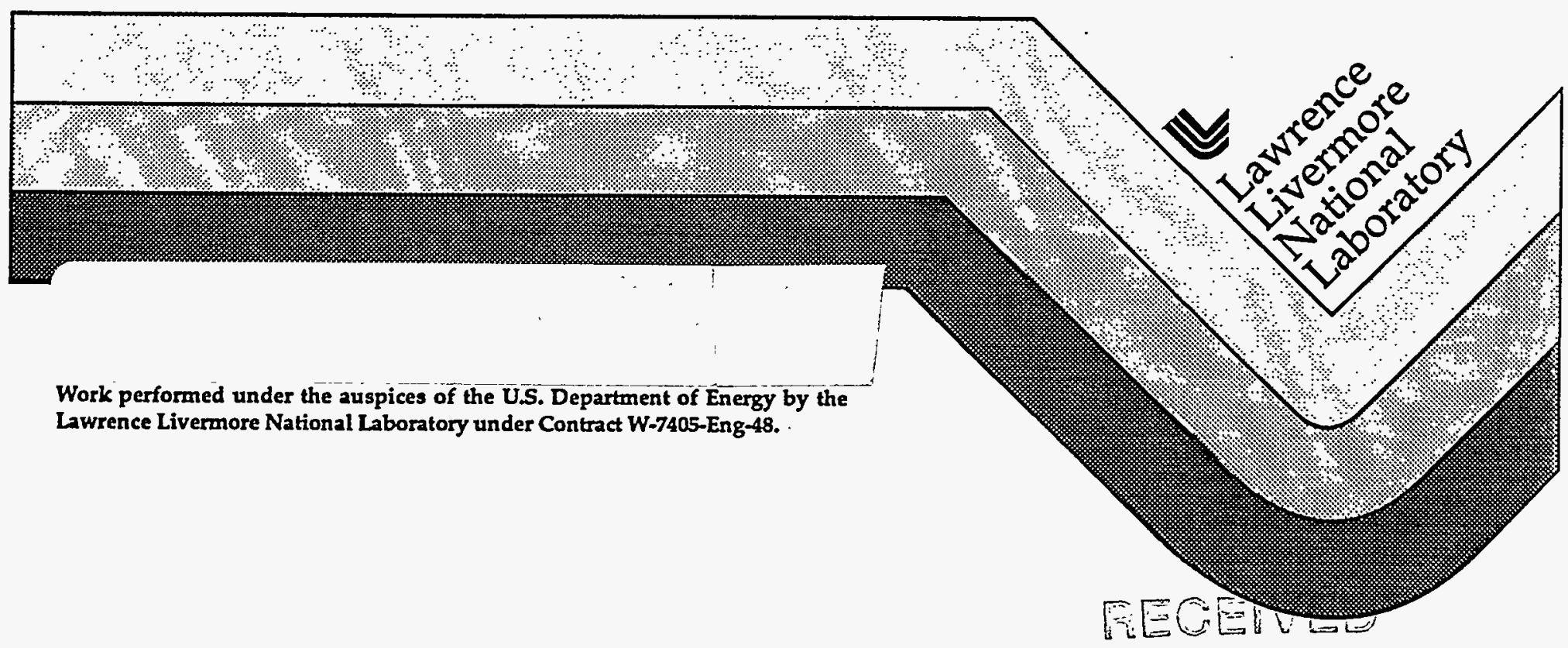

JUL 18 SPSO

OSTI 


\section{DISCLAIMER}

This document was prepared as an account of work sponsored by an agency of the United States Covernment. Neither the United States Covernment not the University of California nor any of their employees, makes any warranty, express or implied, or assumes any legal liability or responsibility for the accuracy, completeness, or usefulness of any information, apparatus, product, or process disclosed, or represents that its use would not infringe privately owred rights. Reference herein to any specific commercial product, process, or service by trade name, trademark, manufacturer, or otherwise, does not necessarily constitute or imply its endorsement, recommendation, or favoring by the United States Government or the University of California. The views and opinions of authors expressed herein do not necessarily state or reflect those of the United States Covernment or the University of California, and shall not be used for advertising or product endorsement purposes.

This report has been reproduced directly from the best available copy.

Available to DOE and DOE contractors from the Office of Scientific and Technical Information P.O. Box 62, Oak Ridge, TN 37831

Prices available from (615) 576-8401, FTS 626-8401

Available to the public from the

National Techrical Information Service

US. Department of Commerce

5285 Port Royal Rd.

Springfield, VA 22161 


\section{DISCLAIMER}

Portions of this document may be illegible in electronic image products. Images are produced from the best available original document. 


\title{
STATUS REPORT ON SOURCE PROPERTIES IMPORTANT FOR DISCRIMINATION*
}

\author{
L. A. Glenn
}

27 March 1995

\section{INTRODUCTION}

To begin, we need to discuss what is meant by source properties important for discrimination. Here we are concerned primarily with discriminating earthquakes from explosions by seismic means. We do not distinguish between chemical and nuclear explosions because, for concentrated explosions, these will appear identical except for a factor of two or so in the long-period amplitude (which will not be identifiable at a distant seismic station). Ripple-fired chemical explosions may, in theory, be distinguished from concentrated explosions by the spectral modulation that is produced by the former, however we specifically exclude spatio-temporal organization and other geometric effects and concentrate on material properties that have important influence on characteristics of the seismic signal which might allow explosions to be distinguished from earthquakes. The most prominent of these are the corner frequency of the seismic spectrum, the high-frequency roll-off, and the long-period overshoot.

Both quasi-analytical ${ }^{1}$ methods and finite difference numerical solutions of the hydrodynamic equations ${ }^{1-6}$ were used to explore the effects of the:

1. equation of state of the explosion products

2. equation of state of the rock media surrounding the explosion

3. energy and mass density of the explosion source

4. constitutive properties of the surrounding rock media, including
a. compressive and tensile strength
b. porosity
c. dilatancy
d. elastic moduli

5. depth of burial (lithostatic overburden)

The calculations performed thus far assumed one-dimensional radial symmetry for the most part so that, in addition to geometric details of the source excluded from consider-

\footnotetext{
* Submitted as FY1995 Scheduled Deliverable No. 8 for LLNL's Regional Seismic Project
} 
ation, depth effects on spall are excluded as well. It is also important to understand that many of the factors itemized above are not single-valued parameters, but rather models for which several parameters normally need to be specified. This is obviously the case for the high-temperature equations of state, where tabular constructions are the most accurate representations, but also for the strength, porosity and dilatancy (which turn out to have much more important influence for seismic discrimination purposes. The models employed are not unique and this further complicates any discussion of source properties. For our analysis we have chosen models that offer a wide range of parametric behavior and also that have generally given good agreement with experiment. It must be admitted, however, that field data are relatively sparce and that models that agree well with laboratory-scale experiments do not always perform well at field scale. The main reason for this is probably the inhomogeneous nature of most rock formations. Model parameters are often set via laboratory analysis of rock cores that have dimension of only a few millimeters whereas field-scale modeling considers elements larger by several orders of magnitude. When the rock properties vary considerably between these scales good agreement in results should not be expected.

\section{MATERIAL MODEIS}

The most important rock material properties are the strength, gas porosity, and possibly the dilatancy. We found that the high-temperature equations of state for both the explosion products and the rock have relatively little influence on the spectral characterisitics of the seismic signal. We generally employed tabular equations of state for both the explosion device and the surrounding rock (at least above the melting point), that were fitted to previously obtained shock Hugoniot and elastic-wave data. The shockwave data are relatively insensitive to small-scale inhomogeneities so that it is generally possible to determine the high-pressure properties of even the rock quite accurately. The elastic moduli are also known accurately via wave speed measurements. By contrast, and as noted above, it is difficult to obtain unambiguous strength or porosity data due mainly to the spatial inhomogeneities inherent at most șites.

In our model, the compressive strength of the rock depends on the pressure, temperature, and on a damage parameter that measures the degree of degradation due to tensile fracture. Specifically,

$$
\sqrt{3 J_{2}^{\prime}} \leq Y=(1-D) \bar{Y}+\beta D \bar{Y}
$$

where $J_{2}^{\prime} \equiv \frac{1}{2} \sigma_{i j}^{\prime} \sigma_{i j}^{\prime}, \sigma^{\prime}$ is the deviatoric stress tensor, $\beta$ is a constant, and $D$ is a scalar function of the volumetric component of the void strain tensor ${ }^{7}$. Tensile fracture is allowed along three orthogonal axes, and cracks are allowed to open and close; once opened, however, the tensile strength normal to the plane of opening is zero. In three 
dimensions, the cracks are free to rotate with the deforming media (with one dimensional radial symmetry, no such rotation is obviously possible) and frictional contact on sliding crack surfaces is allowed. Typically, $D=\epsilon_{i i}^{V} / \epsilon_{\max }^{V}$ and $\epsilon_{\max }^{V}$ is assumed to be on the order of $10^{-2}$. Also,

$$
\bar{Y}=\left[Y_{0}+\frac{p}{a+\left(\frac{p}{Y_{u}-Y_{0}}\right)}\right] \max \left\{\left(1-T / T_{m}\right)^{b}, 0\right\}
$$

$Y_{0}$ is the unconfined compressive strength, $Y_{u}$ is the ultimate compressive strength, $T$ is temperature, and $T_{m}(p)$ is the melting point.

At low pressures, and for generally porous rocks, the scalar part of the stress tensor is based on the well-known $p-\alpha$ concept $^{8}$ :

$$
p(\nu, e)=p_{m}(\nu / \alpha, e) / \alpha=p_{m}\left(\nu_{m}, e_{m}\right) / \alpha
$$

and

$$
\alpha=\alpha(p)
$$

where $\nu=1 / \rho$ is the specific volume, and the subscript $m$ refers to the matrix material in which the pores are embedded. For efficient solution we have employed a quadratic form of the Grüneisen equation for $p_{m}$ in (3) and a piecewise linear representation of the "crush curve" (4). The parameter $\alpha$ is related to the gas porosity, $\Psi$, and is defined by the relation

$$
\alpha \equiv \nu / \nu_{m}=\rho_{m} / \rho=(1-\Psi)^{-1}
$$

At ambient pressure, $\alpha=\alpha^{0}$, the initial value, and at some high pressure, $p_{c}$, the pores will be completely crushed and $\alpha\left(p_{c}\right)=1$. For $p>p_{c}$, the Grüneisen form, $p_{m}$, employed in equation (3) smoothly transitions into the tabular form $p_{m}(\rho, T)$. No specific representation was made for saturated or partially saturated porous media other than to adjust the tabular equation of state and Grüneisen parameters accordingly, assuming a homogeneous mixture of rock and water, and to reduce the strength in accordance with laboratory data. Effective stress theories have sometimes been used to deal with saturated rock media, but we have not found these to provide any better agreement with experiment.

Equations (3) and (4) apply to loading for $p<p_{c}$, but to unloading only when $p<p_{e l}$ i in this small, but important, range the pore volume behaves in strictly elastic fashion. In the range $p_{e l} \leq p<p_{c}$, unloading occurs at constant gas porosity.

The results obtained with this model were described in detail when applied to an analysis of the Non-Proliferation Experiment(NPE) and a nearby nuclear explosion ${ }^{5}$. Numerous simulations were made and compared with experiment. We found that the 
asymptotic (long period) value of the reduced displacement potential, $\phi_{\infty}$, for explosions with the ANFO blasting agent used in the NPE, was larger than that derived for a tamped nuclear explosion of the same yield by a factor of 1.9, in good agreement with the experimental results derived from free-field particle velocity measurements, and also with $m_{b}\left(\mathrm{P}_{n}\right)$ data from the Livermore Nevada Network (LNN). Beyond the corner frequency, the spectra calculated for the chemical and nuclear explosions were indistinguishable, also in good agreement with experiment. It was found possible to match the spectral characteristics by varying both the yield strength and the gas porosity, but the strength required to obtain this match was considerably less than measured in laboratory experiments with small cores pulled from the vicinity of the emplacement. As noted above, however, previous experience has shown that laboratory measurements on core-sized rock samples may not be representative of the extant rock mass from which the samples derive.

Further studies with this model have pointed out some difficulties ${ }^{9}$. We have found that the amplitude of the long-period seismic signal is extremely sensitive to the value assigned to $p_{e l}$, an unfortunate circumstance since this parameter is normally not very well defined by experiment. Another problem with this standard form of the $p-\alpha$ model is the discontinuous unloading modulus that derives near the point $\left(p_{e l}, \alpha_{e l}\right)$. For $p_{e l}-\delta p_{e l}$, where $\delta p_{e l} \rightarrow 0$, unloading occurs at the bulk sound speed in the in situ porous medium, whereas for $p_{e l}+\delta p_{e l}$, and because $\alpha$ remains constant in this region, the unloading bulk sound speed turns out to be very nearly that of the fully compacted medium. The effect of this artifact is to significantly reduce the long-period amplitude of the reduced displacement potential and to thereby induce an artificial overshoot in the Fourier spectrum. When the model was modified to allow smooth transition of unloading bulk moduli from the fully porous to the fully compacted states, the artificial overshoot disappeared.

Laboratory experiments on porous rock samples that are repeatedly loaded and unloaded, at various stress levels in the partially compacted state, exhibit much more complex unloading behavior than the simple models described above. We found that ad hoc representations of this behavior had a significant influence on the shape of the seismic spectrum, especially at long periods. These shape factors may be important for discrimination purposes, so we are planning to implement a physically based porosity model that includes more accurate representation of the unloading/reloading process and also better accounts for effects of partial saturation.

Finally, we should remark on some recent work done in collaboration with the Moscow Engineering Physics Institute ${ }^{10}$ under the auspices of the International Science and Technology Center. This work describes a model of brittle failure in which the influence of dilatancy plays an important role. Normally, the effect of increasing the compressive strength of rock is to reduce the amplitude of the seismic signal. For granitic rocks, 
where inelastic dilatancies of up to $20 \%$ have been observed dynamically, increasing the strength can be shown to increase the amplitude. Although this behavior appears counter-intuitive, recall that the maximum amplitude of the reduced displacement potential is proportional to the change in volume in the inelastic region induced by the explosion. Large dilatancies can therefore overcompensate for the increasing strength, with the net effect being increased seismic amplitude. Additional work is required to provide experimental confirmation, and to examine the effects of dilatancy on the spectral shape.

\section{REFERENCES}

1. Glenn, L. A. Energy-density effects on seismic decoupling, J. Geophys. Res., 98, B2, 1933-1942, 1993.

2. Glenn, L. A. Strain hardening in salt - results of the SALMON experiment, J. Energy Res. Tech. (ASME), 112, 145-148, 1990.

3. Glenn, L. A. Comparing U. S. and Russian experience with cavity decoupling in salt, Geophys. Res. Lett., 20, No. 10, 919-922, 1993.

4. Glenn, L. A. and P. Goldstein. Seismic decoupling with chemical and nuclear explosions in salt, J. Geophy. Res., 99, B6, 11723-11730, 1994.

5. Glenn, L. A. and P. Goldstein. The influence of material models on chemical or nuclear-explosion source function, Proceedings of the DOE Symposium on the NonProliferation Experiment Results and Implications for Test Ban Treaties, April 19-21 1994 (see also UCRL-JC-116428, April 1994).

6. Glenn, L. A. KUCHEN: An experiment to evaluate decoupling in high-aspect-ratio cavities, Proceedings of the DNA/ACDA VERTECH II Symposium, Washington, D. C., January 10-12, 1995 (see also UCRL-JC-119103, January 1995).

7. Rubin, M. B. and A. V. Attia. A continuum tensile failure model with friction, UCRLID-104759, August 21, 1990.

8. Carroll, M. M. and A. C. Holt. Suggested modification of the $p-\alpha$ model for porous materials, J. Appl. Phys., 43, No. 2, 759-761, 1972.

9. Glenn, L. A. Modeling effects of gas porosity on simulations of seismic source functions, manuscript in preparation.

10. Bobryshev, V. V., V. S. Fetisov, V. K. Sirotkin and L. A. Glenn. A model of the seismic source function for underground explosions in plastic dilatant media, manuscript in preparation. 